\title{
MIR298 Pre-miRNA
}

National Cancer Institute

\section{Source}

National Cancer Institute. MIR298 Pre-miRNA. NCI Thesaurus. Code C82816.

MIR298 is an oligoribonucleotide that is encoded by the human MIR298 gene and has a role in the regulation of gene expression. 